\title{
The vaginal microbiota in the course of bacterial vaginosis treatment
}

\author{
Romy D. Zwittink ${ }^{1,2}$ (D) Ellen H. A. van den Munckhof ${ }^{2,3} \cdot$ Maurine A. Leverstein-van Hall $^{3,4} \cdot$ Kim Boers $^{5}$. \\ Anco Molijn ${ }^{3} \cdot$ Cornelis W. Knetsch $^{3} \cdot$ Ed J. Kuijper $^{1,2}$
}

Received: 20 April 2020 / Accepted: 24 September 2020 / Published online: 7 October 2020

(C) The Author(s) 2020

\begin{abstract}
Bacterial vaginosis (BV) is perceived as a condition of disrupted vaginal microbiota, but remains of unknown aetiology. In this study, vaginal microbiota composition was determined in twenty-one women with $\mathrm{BV}$, before and after treatment with metronidazole or clindamycin. Microbiota composition varied greatly between women and defining a (un)healthy vaginal microbiota state remains elusive, challenging BV diagnosis and treatment. While relative abundance of Lactobacillus increased after antibiotic treatment in two-third of women, its abundance was not associated with treatment outcome. Instead, remaining complaints of abnormal vaginal discharge were more common after metronidazole treatment and associated with increased relative abundance of Ureaplasma.
\end{abstract}

Keywords Antibiotics $\cdot$ Bacterial vaginosis $\cdot$ Clindamycin $\cdot$ Metronidazole $\cdot$ Microbiota $\cdot 16 \mathrm{~S}$ rRNA gene amplicon sequencing

\section{Introduction}

The vaginal microbiota plays a crucial role in maintaining a healthy vaginal environment, and perturbation of this system has been implicated in disturbed vaginal health and other negative outcomes $[1,2]$. The vaginal microbiota is dynamic and influenced by hormonal changes, sexual activity, and hygiene [3]. Various vaginal bacterial communities exist in healthy women, mostly dominated by Lactobacillus species, while some are being composed of anaerobes like Atopobium and Prevotella species [4]. Nevertheless, the common perception of a healthy vaginal microbiota is one dominated by one or

Romy D. Zwittink and Ellen H. A. van den Munckhof contributed equally to this work.

Romy D. Zwittink

r.d.zwittink@1umc.nl

1 Center for Microbiome Analyses and Therapeutics, Leiden University Medical Center, Leiden, The Netherlands

2 Department of Medical Microbiology, Leiden University Medical Center, Albinusdreef 2, 2333, ZA Leiden, The Netherlands

3 DDL Diagnostic Laboratory, Rijswijk, The Netherlands

4 Department of Medical Microbiology, Alrijne Hospital, Leiden, The Netherlands

5 Department of Gynaecology, Haaglanden Medical Centre, The Hague, The Netherlands more Lactobacillus species. As such, the switch from a Lactobacillus-dominated microbiota to a more diverse microbiota, in combination with clinical symptoms, is considered bacterial vaginosis (BV) or aerobic vaginitis, depending on colonisation by anaerobic or aerobic bacteria, respectively. Bacterial genera that are specifically associated with bacterial vaginosis are, amongst others, Gardnerella, Atopobium, Prevotella, Fusobacterium, and Dialister species [5]. Despite these associations, the aetiology of BV is unknown, and diagnosis and treatment remain elusive. While a Gramstain evaluation according to the Nugent criteria is considered the golden standard for BV diagnosis, it is not routinely applied in a clinical setting [6]. Instead, BV diagnosis is commonly based on clinical signs and symptoms or Amsel criteria [7]. Symptoms of BV can be resolved without intervention, but metronidazole or clindamycin can be prescribed in case of persistence, even though recurrence is common $[8,9]$. In our study, vaginal microbiota composition of women with clinically diagnosed $\mathrm{BV}$ was determined before and after antibiotic treatment and related to clinical characteristics.

\section{Materials and methods}

Prospectively, vaginal secretions and clinical data were collected from 60 premenopausal women visiting the gynaecology outpatient clinic of the Haaglanden Medical Centre (The 
Hague, The Netherlands) with complaints of abnormal vaginal discharge. Vaginal secretion was collected using the ESwab (Copan Diagnostics Inc., USA). BV diagnosis was based on clinical symptoms and signs, with malodorous discharge, as major criteria for diagnosis of bacterial vaginosis, followed by culturing when clinical diagnosis based on symptoms alone was uncertain. Therapy was initiated according to routine hospital practice following the European guideline and consisted of $500 \mathrm{mg}$ metronidazole taken orally twice a day for 7 days, or, in case of pregnancy or lactating, $300 \mathrm{mg}$ clindamycin taken orally twice a day for 7 days [10]. A follow-up visit was scheduled approximately 4 weeks after inclusion, during which vaginal swab and clinical data collection were repeated. Women who were clinically diagnosed with BV and attended the follow-up visit were selected for microbiota profiling $(n=$ 21). Clinical data collection, Amsel criteria (vaginal $\mathrm{pH}$, amine odour, wet-mount microscopy), Nugent score, and Gardnerella vaginalis culturing were performed for research purposes as previously described [11]. Detailed subject characteristics are outlined in Table 1. The Declaration of Helsinki was the guiding principle for trial execution, and the study was approved by the local ethics board (METC Zuidwest Holland, The Hague, The Netherlands). All patients provided written informed consent before participation.

Vaginal bacterial microbiota was determined by $16 \mathrm{~S}$ rRNA gene amplicon sequencing of the V3-V4 region using the Nextera XT, MiSeq Reagent Kits v2 500 cycles, and a MiSeq desktop sequencer (Illumina, USA). Raw sequencing data are available in the NCBI Sequence Read Archive (https://www.ncbi.nlm.nih.gov/sra) under study accession PRJNA524112. Read filtering, operational taxonomic unit (OTU)-picking, and taxonomic assignment were performed using the NGTax 0.4 pipeline and the Silva_132_SSU Ref database [12]. Statistical analysis and data visualisation were performed in R (v3.5.1) using the packages phyloseq (v1.26. 1), vegan (v2.5-4), ggplot2 (v3.1.0), DESeq2 (v1.22.2) microbiome (v1.4.2), and DirichletMultinomial (v1.24. 1). For differential abundance testing by DESeq2, the OTU-table was filtered for OTUs present in less than $25 \%$ of the samples to minimise zero-variance errors and spurious significance. Permutational multivariate analysis of variance was performed using the adonis function with 999 permutations and Bray-Curtis distances to determine associations between microbiota composition and clinical variables. The Dirichlet Multinomial Mixtures method, using the Laplace equation, was applied for community typing. In this approach, samples are clustered based on microbiota profile similarity [13]. Kruskal-Wallis followed by post hoc Dunn's testing was performed to compare Shannon diversity indices between groups.
Table 1 Subject characteristics

\begin{tabular}{|c|c|c|}
\hline & Before treatment & After treatment \\
\hline \multicolumn{3}{|l|}{ Demographics } \\
\hline$n$ & 21 & 21 \\
\hline Age (years) & $32.5 \pm 7.6$ & $32.5 \pm 7.6$ \\
\hline European & 15 & 15 \\
\hline \multicolumn{3}{|l|}{ Antimicrobials } \\
\hline Clindamycin & - & 11 \\
\hline Metronidazole & - & 10 \\
\hline Clotrimazole & - & 4 \\
\hline Azithromycin & - & 1 \\
\hline \multicolumn{3}{|l|}{ Symptomology } \\
\hline Abnormal discharge & 21 & 9 \\
\hline Malodorous discharge & 20 & 4 \\
\hline Increased discharge & 13 & 5 \\
\hline Yellow/green discharge & 7 & 2 \\
\hline Curdy discharge & 2 & 2 \\
\hline Thin white discharge & 8 & 5 \\
\hline Purulent discharge & 1 & 0 \\
\hline Vulvar erythema oedema & 4 & 2 \\
\hline Vulvar itching & 9 & 3 \\
\hline Vulvar irritation & 6 & 3 \\
\hline Cervical erythema & 3 & 2 \\
\hline Cervical bleeding & 1 & 0 \\
\hline Low abdominal pain & 10 & 3 \\
\hline \multicolumn{3}{|l|}{ Diagnosis } \\
\hline Bacterial vaginosis & 21 & 2 \\
\hline Nugent score positive & 12 & 5 \\
\hline Amsel criteria positive & 13 & 4 \\
\hline Vaginal $\mathrm{pH}>4.5$ & 18 & 12 \\
\hline Amine odour & 16 & 8 \\
\hline Clue cells & 14 & 4 \\
\hline \multicolumn{3}{|l|}{ Other } \\
\hline Anticonception & 6 & 6 \\
\hline Vaginal shower gel & 0 & 1 \\
\hline Sexually active & 20 & 20 \\
\hline Pregnant & 8 & 8 \\
\hline Lactating & 3 & 3 \\
\hline PROM & 0 & 0 \\
\hline
\end{tabular}

Table 2 Core microbiota before and after antibiotic treatment. Bacterial taxa were considered part of the core microbiota when present in $75 \%$ of the samples from the specified group

\begin{tabular}{ll}
\hline Bacterial genus & Average relative abundance (fraction) \\
\hline Before treatment & \\
Gardnerella & 0.294 \\
Atopobium & 0.104 \\
Prevotella & 0.132 \\
Lactobacillus & 0.151 \\
Dialister & 0.038 \\
After treatment & \\
Lactobacillus & 0.608 \\
\hline
\end{tabular}


Table 3 Clinical variables significantly associated with microbiota composition before and after antibiotic treatment

\begin{tabular}{lll}
\hline Variable & $R^{2}$ & $p$ value \\
\hline Before treatment & & \\
Nugent score & 0.238 & 0.001 \\
Anticonception & 0.146 & 0.008 \\
Lactating & 0.091 & 0.008 \\
pH $>4.5$ & 0.086 & 0.012 \\
Amine odour & 0.073 & 0.028 \\
After treatment & & \\
& & \\
Nugent score & 0.499 & 0.001 \\
pH $>4.5$ & 0.143 & 0.006 \\
\hline
\end{tabular}

\section{Results and discussion}

Before antibiotic treatment, genera Gardnerella, Atopobium, Prevotella, Lactobacillus, and Dialister constituted the core microbiota, and combined accounted for an average relative abundance of $71.9 \%$ (Table 2), but their abundance could vary greatly between subjects (Fig. 1a). Two community types could be identified, one driven by Gardnerella, Prevotella, Sneathia, and Atopobium (community type 1), and one driven by Lactobacillus, Gardnerella, and Atopobium (community type 2, Fig. 2a), suggesting Lactobacillus, Prevotella, and Sneathia abundances as discriminative feature of microbiota composition between patients. Bacterial diversity significantly differed between the two community types (Fig. 3a), with lower diversity in the Lactobacillus-driven community type. Microbiota composition before treatment was significantly associated with various parameters (Table 3), including the Nugent score, hormonerelated variables (lactation, anticonception use), and BV symptomology (vaginal $\mathrm{pH}$ and amine odour).

After treatment, bacterial diversity decreased (Fig. 3c) and the core microbiota solely consisted of Lactobacillus, constituting an average of $60.8 \%$ relative abundance (Table 2). a Before treatment
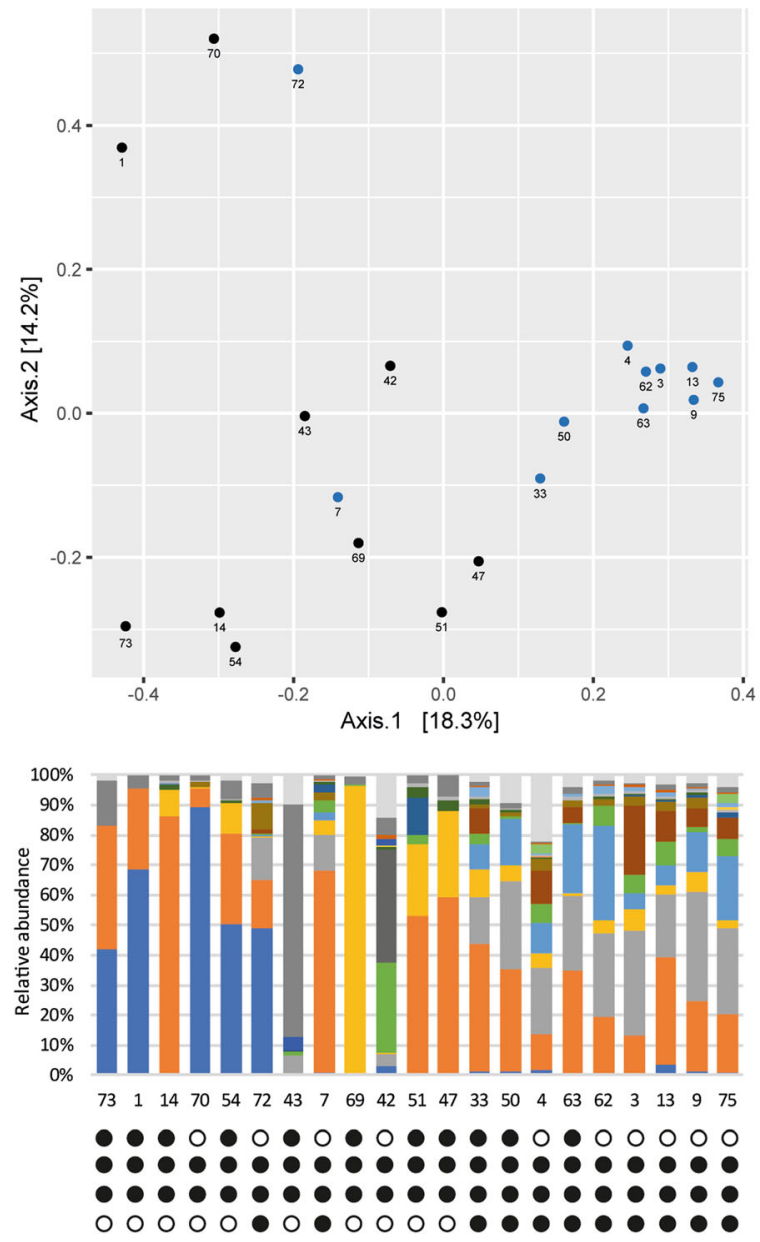

Fig. 1 Principal coordinate analysis and taxonomic profiles of the vaginal microbiota before (a) and after (b) antibiotic treatment. Numbers indicate individual patients. Twenty taxa with highest average relative abundance are shown; abundances of all other taxa are summed and categorised as

\section{b After treatment}

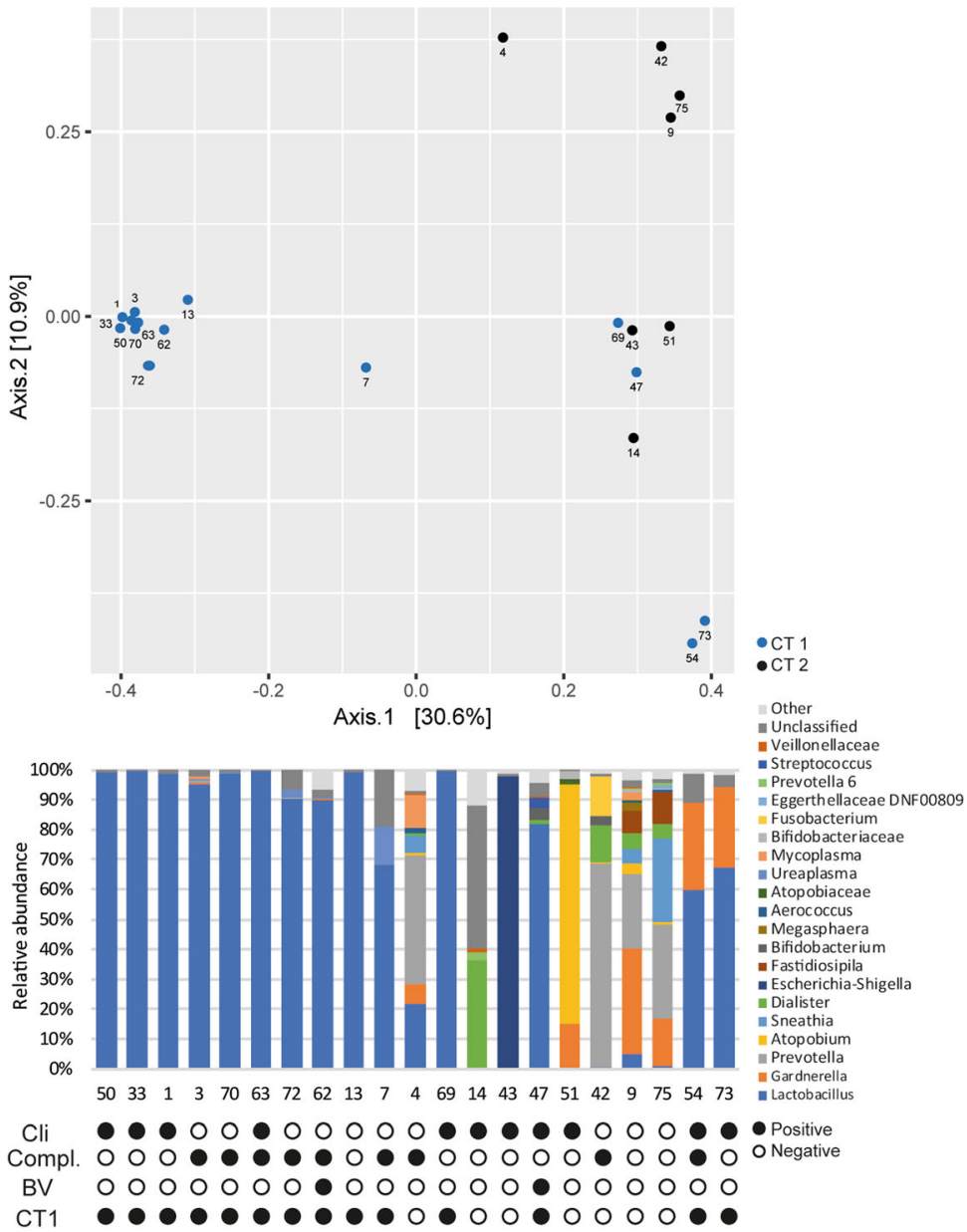

'other'. For bar graphs, the subject order is matched to the subject order in the PCoA plots. Cli, clindamycin; Compl., complaints of abnormal vaginal discharge; CT1, community type one; CT2, community type 2 

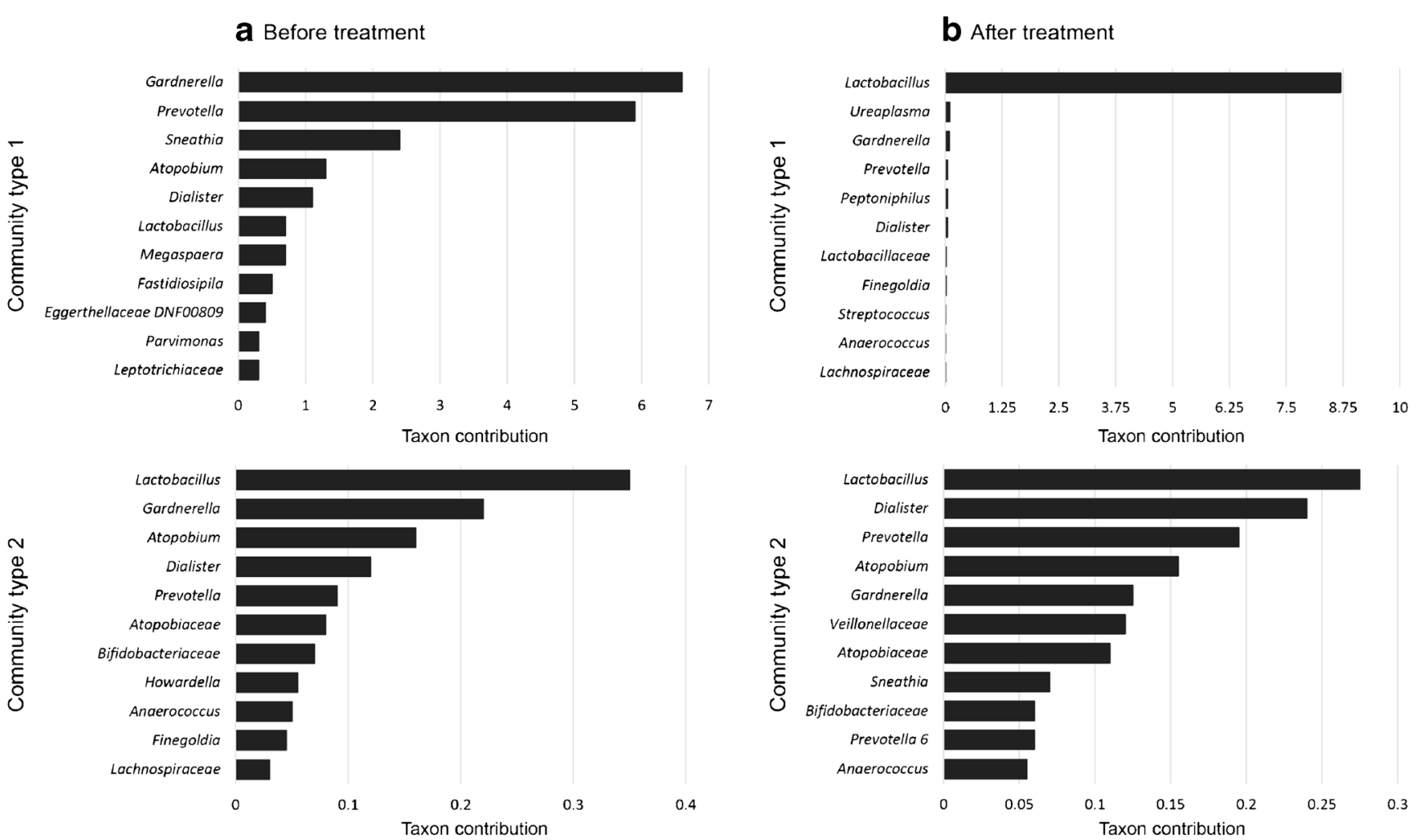

Fig. 2 Vaginal microbiota community types before (a) and after (b) antibiotic treatment. For each community type, the 11 main driving bacterial taxa are shown

Independent of antibiotic type (metronidazole or clindamycin), antibiotic treatment significantly decreased the relative abundance of Atopobium $(\log 2$ FoldChange $=-3.36$, padj $=0.0388)$, while increasing Lactobacillus $(\log 2$ FoldChange $=4.04$, padj $=0.0002)$. However, Lactobacillus remained of low abundance in one-third of the women, whose microbiota was of individual-specific composition with high abundance of either Gardnerella, Prevotella, Dialister, Escherichia-Shigella, Atopobium, or Sneathia (Fig. 1b). These microbiota compositions were also reflected by the identification of two community types: one driven by Lactobacillus, and the other driven by multiple bacterial taxa (Fig. 2b), with lower diversity in the Lactobacillus-driven community type (community type 1, Fig. 3b). Vaginal microbiota composition after antibiotic treatment was significantly associated with the Nugent score and vaginal $\mathrm{pH}$ (Table 3).

These findings support the current debate on the definition of a healthy vaginal microbiota [14], since Lactobacillus dominance was observed in a large proportion of women with symptoms and the opposite, dominance of anaerobes, was observed in asymptomatic women. So even in a study of small subject size, as herein, heterogeneity of vaginal bacterial communities was apparent. Vaginal health status may be associated with specific Lactobacillus species [10], which could not be defined by the method used herein. However, several kinds of microbiota composition existed in asymptomatic women, which has been previously reported $[4,11,15]$. Vaginal microbiota composition was consistently associated with the Nugent score and vaginal $\mathrm{pH}$. While the Nugent score is considered the golden standard for BV diagnosis, it is rarely used in clinical setting due to resource intensiveness [6]. Determining vaginal $\mathrm{pH}$ is more readily applicable; however, it most certainly simply reflects the abundance of lactic acid-producing bacteria, like Lactobacillus. Nowadays, PCR-based laboratory tests would be preferred for confirmation of the diagnosis [16]. Except lactation and anticonception use, vaginal microbiota composition was not associated with patient demographics and lifestyle factors, which may be due to the relatively small subject size in combination with uniformity. It has previously been reported that host genetics, ethnicity, hormonal stage (e.g. menstruation cycle, menopause, pregnancy), sexual behaviour, and hygiene practices, amongst others factors, influence vaginal microbiota composition [17-21].

After antibiotic treatment, nine women $(43 \%)$ reported remaining complaints of abnormal vaginal discharge. Persisting complaints were more prevalent in women receiving metronidazole $(70 \%)$ than in those receiving clindamycin (18\%), which may be a result of differences in antibiotic spectrum and underlying conditions (e.g. pregnancy). To determine the potential influence of the microbiota on clinical outcome, vaginal microbiota composition before and/or after treatment were compared between patients with and without persistent complaints. The vaginal microbiota of women with persisting complaints contained a significantly higher relative abundance of 
a Before treatment

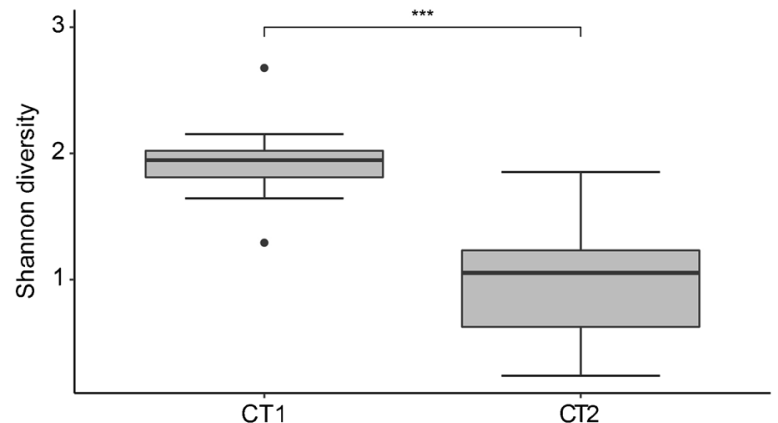

b After treatment

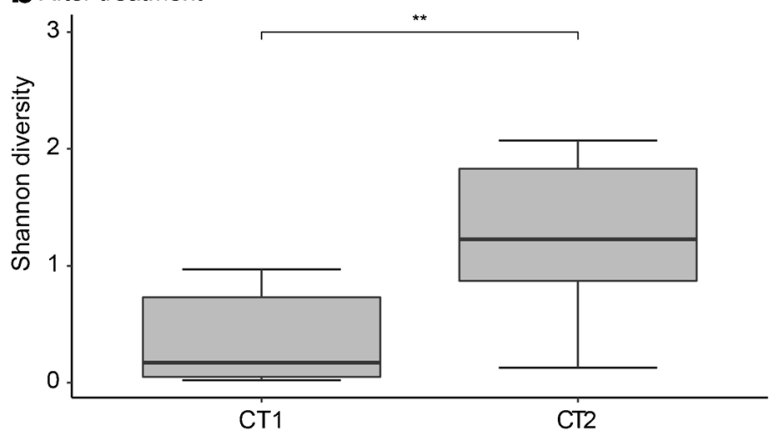

C Before versus after treatment

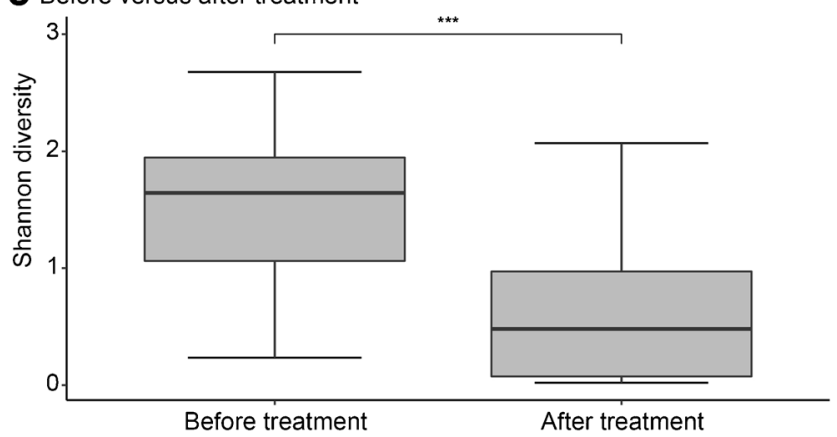

Fig. 3 Bacterial diversity of each community type before treatment (a) and after treatment (b), and of all samples before and after treatment (c). Boxplots indicate the median, 25th and 75th percentile and whiskers indicate $1.5 *$ interquartile range. $* p<0.05, * * p<0.01, * * * p<0.001$. $\mathrm{CT}$, community type

Ureaplasma $(\log 2$ FoldChange $=8.73$, pajd $=0.0008)$, but persisting complaints could not be associated with microbiota composition before treatment. Ureaplasma is a parasitic and saprophytic bacterium belonging to the Mollicutes class and is without cell wall, which results in intrinsic resistance to cell walltargeting antibiotics like beta-lactam and glycopeptide antibiotics [22]. Ureaplasma is intrinsically resistant to metronidazole, but usually susceptible to clindamycin [23]. While carriage of Ureaplasma in the urethra, cervix, and vagina is common and generally asymptomatic, it has previously been associated with BV recurrence [24]. Treatment outcome was not associated with the identified community types after treatment as persistent complaints were reported in 50\% (7/14) and 29\% (2/7) of women with vaginal microbiota composition belonging to the Lactobacillus-driven community type one or multiple speciesdriven community type two, respectively.

\section{Conclusion}

In conclusion, defining a (un)healthy vaginal microbiota state remains elusive, which challenges diagnosis and treatment of $\mathrm{BV}$. Abnormal vaginal discharge and itching/irritation is most certainly not attributable to one or more specific bacteria, rather a disruption of the individual-specific mutualistic relationship of bacterial communities. Nevertheless, establishing universal markers for diagnosis and treatment of BV remains relevant. Herein, remaining complaints after treatment were more common in women who received metronidazole and were associated with increased relative abundance of the Ureaplasma genus, which may be considered when treatment fails.

Authors' contributions RZ performed microbiome analyses, interpreted data, made figures, and wrote drafts of the manuscript. EM designed the study, performed NGS, interpreted data, made the subjects table, and revised drafts of the manuscript. ML, KB, and AM designed the study and revised drafts of the manuscript. CK and EK supervised RZ and EM and interpreted data and revised drafts of the manuscript.

Data availability Raw sequencing data are available in the NCBI Sequence Read Archive (https://www.ncbi.nlm.nih.gov/sra) under study accession PRJNA524112.

\section{Compliance with ethical standards}

Conflict of interest EK is supported by an unrestricted grant from Vedanta Biosciences Inc. and has performed research for Cubist, Novartis, and Qiagen, and has participated in advisory forums of Astellas, Optimer, Actelion, Pfizer, Sanofi Pasteur, and Seres Therapeutics. These companies had no role in the research described in this manuscript. All other authors declare that they have no conflict of interest.

Ethics approval The study was approved by the local medical ethics board (METC Zuidwest Holland, The Hague, The Netherlands).

Consent to participate Written informed consent was obtained from all participating subjects.

Consent for publication Written informed consent was obtained from all participating subjects.

Code availability Raw sequencing data was analysed using free, open source software NG-Tax (http://wurssb.gitlab.io/ngtax/) and R (https:// www.r-project.org/).

Open Access This article is licensed under a Creative Commons Attribution 4.0 International License, which permits use, sharing, adaptation, distribution and reproduction in any medium or format, as long as you give appropriate credit to the original author(s) and the source, provide a link to the Creative Commons licence, and indicate if changes were made. The images or other third party material in this article are included in the article's Creative Commons licence, unless indicated otherwise in a credit line to the material. If material is not included in the article's Creative Commons licence and your intended use is not permitted by statutory regulation or exceeds the permitted use, you will need to obtain 
permission directly from the copyright holder. To view a copy of this licence, visit http://creativecommons.org/licenses/by/4.0/.

\section{References}

1. Greenbaum S, Greenbaum G, Moran-Gilad J, Weintraub AY (2019) Ecological dynamics of the vaginal microbiome in relation to health and disease. Am J Obstet Gynecol 220(4):324-335

2. Kroon SJ, Ravel J, Huston WM (2018) Cervicovaginal microbiota, women's health, and reproductive outcomes. Fertil Steril 110(3): 327-336

3. Huang B, Fettweis JM, Brooks JP, Jefferson KK, Buck GA (2014) The changing landscape of the vaginal microbiome. Clin Lab Med 34(4):747-761

4. Ma B, Forney LJ, Ravel J (2012) Vaginal microbiome: rethinking health and disease. Annu Rev Microbiol 66:371-389

5. Onderdonk AB, Delaney ML, Fichorova RN (2016) The human microbiome during bacterial vaginosis. Clin Microbiol Rev 29(2): 223-238

6. Bagnall P, Rizzolo D (2017) Bacterial vaginosis: a practical review. JAAPA 30(12): 15-21

7. Amsel R, Totten PA, Spiegel CA, Chen KC, Eschenbach D, Holmes KK (1983) Nonspecific vaginitis. Diagnostic criteria and microbial and epidemiologic associations. Am J Med 74(1):14-22

8. Bradshaw CS, Morton AN, Hocking J et al (2006) High recurrence rates of bacterial vaginosis over the course of 12 months after oral metronidazole therapy and factors associated with recurrence. $\mathrm{J}$ Infect Dis 193(11):1478-1486

9. Koumans EH, Markowitz LE, Hogan V, Group CBW (2002) Indications for therapy and treatment recommendations for bacterial vaginosis in nonpregnant and pregnant women: a synthesis of data. Clin Infect Dis 35(Suppl 2):S152-S172

10. Wang B, Xiao BB, Shang CG et al (2014) Molecular analysis of the relationship between specific vaginal bacteria and bacterial vaginosis metronidazole therapy failure. Eur J Clin Microbiol Infect Dis 33(10):1749-1756

11. Albert AY, Chaban B, Wagner EC et al (2015) A study of the vaginal microbiome in healthy Canadian women utilizing cpn60based molecular profiling reveals distinct Gardnerella subgroup community state types. PLoS One 10(8):e0135620

12. Ducarmon QR, Hornung BVH, Geelen AR, Kuijper EJ, Zwittink RD (2020) Toward standards in clinical microbiota studies: comparison of three DNA extraction methods and two bioinformatic pipelines. mSystems 5(1)

13. Arumugam M, Raes J, Pelletier E et al (2011) Enterotypes of the human gut microbiome. Nature 473(7346):174-180

14. Reid G (2018) Is bacterial vaginosis a disease? Appl Microbiol Biotechnol 102(2):553-558

15. Anahtar MN, Byrne EH, Doherty KE et al (2015) Cervicovaginal bacteria are a major modulator of host inflammatory responses in the female genital tract. Immunity 42(5):965-976

16. van den Munckhof EHA, van Sitter RL, Boers KE et al (2019) Comparison of Amsel criteria, Nugent score, culture and two CEIVD marked quantitative real-time PCRs with microbiota analysis for the diagnosis of bacterial vaginosis. Eur J Clin Microbiol Infect Dis 38(5):959-966

17. Mehta SD, Nannini DR, Otieno F et al (2020) Host Genetic factors associated with vaginal microbiome composition in Kenyan women. mSystems 5(4)

18. Xu J, Bian G, Zheng M et al (2020) Fertility factors affect the vaginal microbiome in women of reproductive age. Am J Reprod Immunol 83(4):e13220

19. Fashemi B, Delaney ML, Onderdonk AB, Fichorova RN (2013) Effects of feminine hygiene products on the vaginal mucosal biome. Microb Ecol Health Dis 24

20. Song SD, Acharya KD, Zhu JE et al (2020) Daily vaginal microbiota fluctuations associated with natural hormonal cycle, contraceptives, diet, and exercise. mSphere 5(4)

21. Noyes N, Cho KC, Ravel J, Forney LJ, Abdo Z (2018) Associations between sexual habits, menstrual hygiene practices, demographics and the vaginal microbiome as revealed by Bayesian network analysis. PLoS One 13(1):e0191625

22. Beeton ML, Spiller OB (2017) Antibiotic resistance among Ureaplasma spp. isolates: cause for concern? J Antimicrob Chemother 72(2):330-337

23. Sweeney EL, Dando SJ, Kallapur SG, Knox CL (2017) The human Ureaplasma species as causative agents of chorioamnionitis. Clin Microbiol Rev 30(1):349-379

24. Xiao B, Wu C, Song W et al (2019) Association analysis on recurrence of bacterial vaginosis revealed microbes and clinical variables important for treatment outcome. Front Cell Infect Microbiol 9:189

Publisher's note Springer Nature remains neutral with regard to jurisdictional claims in published maps and institutional affiliations. 